\title{
Aprendizajes digitales construidos junto a niñas y niños en situación de segregación escolar socioeconómica
}

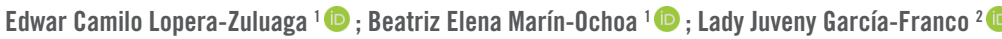 \\ ${ }^{1}$ Universidad Pontificia Bolivariana (UPB), Colombia; ${ }^{2}$ Secretaría de Educación Bello, Colombia.
}

Resumen. Las profesoras y los profesores en Latinoamérica vivimos entre la potencia de la escuela digital y, al tiempo, la actualidad de la segregación escolar socioeconómica de nuestros estudiantes. Por tanto, preguntamos: ¿cómo estimular aprendizajes digitales a pesar de la situación de segregación escolar socioeconómica?, al definirse, ¿qué aprendizajes logran estimular a los niños y niñas? Un profesor investigador y 71 niñas y niños entre 9-13 años construimos una investigación acción, que consistió en el uso de una estrategia digital en pensamiento computacional, además, de la ejecución de talleres de clase, grupos focales y una recuperación familiar de la experiencia vivida para capitalizar los aprendizajes construidos. Empleamos un análisis cualitativo asistido por el software Atlas ti -versión 8-. Fundamentamos en los datos ocho aprendizajes construidos por las niñas y niños: aprendizajes espontáneos; censura contenidos; enseñanza a la familia; identificación de los propios aprendizajes; pertenencia a grupos de interés en línea: habilidad para jugar; sentimientos frente al aprendizaje; y, utilidad escolar de la tecnología. Al final discutimos los resultados en el marco de los antecedentes investigativos, sus implicaciones metodológicas y sus prácticas.

Palabras clave: educación tecnológica; escuelas marginadas; estudiantes de escuela primaria; estrategias de aprendizaje; investigación acción; profesor investigador.

Aprendizado digital construído com crianças em situação de segregação socioeconômica e escolar Resumo. . Nós, professores e professoras da América Latina, vivemos entre o potencial da escola digital e o momento atual de segregação socioeconômica e escolar de nossos alunos. Portanto, perguntamos: como podemos estimular o aprendizado digital, apesar da situação de segregação socioeconômica e escolar? Que conhecimentos conseguem estimular as crianças? Como professor pesquisador, construí com 71 meninos e meninas, com idades entre 9-13anos, uma pesquisa-ação, que consistiu no uso de uma estratégia digital em pensamento computacional, além da realização de oficinas em sala de aula, grupos focais e uma recuperação familiar da experiência vivida para capitalizar o aprendizado construído. Realizamos uma análise de dados qualitativos assistida pelo software Atlas. ti 8. Oito lições aprendidas pelas meninas e meninos foram fundamentadas nos dados: aprendizagem espontânea, censura de conteúdo, ensino à família, identificação de seu próprio aprendizado; pertencimento a grupos de interesse on-line: capacidade de brincar, sentimentos frente a aprendizagem e a utilidade da tecnologia na escola. No final, discutimos os resultados de acordo com os antecedentes da pesquisa, suas implicações metodológicas e suas práticas.

Palavras-chave: educação tecnológica; escolas marginalizadas; estudantes do ensino fundamental/ básico; estratégias de aprendizagem; pesquisa-ação; professor pesquisador.

Digital learnings built with children in situation of school socioeconomic segregation

Abstract. We, teachers in Latin America, live between the potential of digital school and, at the same time, the actual situation of school socioeconomic segregation of our students. Therefore, we ask: how to build digital learning despite the situation of socioeconomic school segregation? When defining itself, what learning can boys and girls build? Both, a teacher researcher and 71 girls and boys between 9-13 years old, built an action research that consisted of the use of a digital strategy in computational thinking, in addition, the execution of class workshops, focus groups and a family recovery of the lived experience to capitalize built learning. We used a qualitative analysis assisted by the Atlas ti software -version 8-. We grounded on data eight learnings built by girls and boys: spontaneous learning; to censor content; to teach the family; to identify their own learning; to belong to online communities; to know how to play; feelings about learning; and, school usefulness of technology. Finally, we discuss the findings within the research background, its methodological and practical implications.

Keywords: Disadvantaged schools; elementary school students; learning strategies; action research; teacher researcher; technology education. 


\section{Introducción}

Hay casi unanimidad en torno al potencial de las tecnologías digitales para el cambio educativo. Al respecto, Selwyn (2016) clasifica este acuerdo en un rango que va desde la modesta idea del mejoramiento hasta la más eufórica sensación de revolución educativa. No obstante, la idea por si misma no deja de ser problemática para la cotidianidad de escuelas públicas segregadas que, en América Latina, configuran uno de los fenómenos educativos menos advertidos, pero con gran responsabilidad en la desigualdad de los aprendizajes entre escuelas ricas y pobres (Krüger, 2019; Murillo, 2019; Murillo y Martínez-Garrido, 2020). Mientras las políticas educativas de cada nación publicitan agendas pro-escuela digital o los aprendizajes necesarios para la sociedad del siglo XXI, las profesoras y los profesores, en la cotidianidad escolar, vemos con frustración una amplia brecha entre la potencia de la escuela digital y la escuela en situación de segregación escolar socioeconómica. De acuerdo con Galtung (1969), observamos con preocupación el violento impacto de esta situación en el potencial nivel de realización educativa de niños y niñas de la región.

Sin embargo, ante las insuficientes condiciones estructurales, no nos queda otra salida que apelar al voluntarismo ferviente o, según Tedesco (2012), hacer de las políticas digitales, universales y homogénas; políticas de subjetividad que devengan en aprendizajes digitales a pesar del contexto de segregación escolar socioeconómica vivido. Entonces, como profesores de escuela primaria nos preguntamos: ¿cómo estimular aprendizajes digitales a pesar de la situación de segregación escolar socioeconómica?, al definirse, ¿qué aprendizajes logran estimular niñas y niños? Las respuestas las ofrecemos en el siguiente artículo a través de tres momentos.

En el primero, haciendo conciencia del problema, a través de una revisión para contrastar el sugerente panorama de la educación digital y el sombrío fenómeno de la segregación escolar socioeconómica en Latinoamérica; contraste, antes que esperanzador, limitador del potencial nivel de realización educativa de niñas y niños. En el segundo momento, ensayamos una salida a la limitación a través de una investigación acción catalizada por una estrategia en pensamiento computacional y capitalizada por talleres en clase, grupos focales y una recuperación familiar de la experiencia vivida, para revelar los aprendizajes construidos. En el tercer momento, presentamos ocho aprendizajes digitales construidos por la acción y la narración de las niñas y los niños. 


\section{Entre potencial y actual, o hacer conciencia de una limitación}

Las prioridades de los sistemas educativos oficiales dejaron de ser una decisión exclusiva de la centralidad gubernamental para transitar a un modelo de gobernanza en red (Ball et al., 2017; Rizvi y Lingard, 2013), integrada por comunidades epistémicas (Haas, 1992) que asesoran, supervisan y se reconocen responsables de los aprendizajes útiles que niñas y niños deben desarrollar para la sociedad del siglo XXI. En esta perspectiva, los aprendizajes digitales conforman el repertorio de los incuestionables para las nuevas políticas educativas (Rivas, 2019a); los saberes sine qua non para la escuela del futuro.

La literatura que fundamenta los aprendizajes digitales es diversa. Profesoras y profesores caminamos, unos abrumados y otros entusiastas, por un paraje de potenciales saberes especializados en alfabetización computacional escolar (British Council, 2020; Nelson et al., 2016), emprendimiento digital, mercadeo y comercio electrónico (Google for education, 2020; Microsoft, 2020), empleos decentes para los jóvenes (ITU, 2019), ciudadanía digital (Carretero et al., 2017), competencias para el mundo digital (CEN, 2020; OCDE, 2019c; SFIA Foundation, 2020; Unesco Institute for Statistics, 2018). Solo una ligera mención a algunos marcos de referencia que, con incisiva publicidad, nos recuerdan que el coeficiente intelectual es cuestión del pasado porque ahora profesoras y profesores caminan en procura del coeficiente de inteligencia digital (DQ Institute, 2019).

Este tránsito es a la vez productivo y utópico. Es productivo porque dota a las niñas y los niños de habilidades útiles para embarcarse en los agudos vectores de la economía digital que prescriben los trabajos del futuro (OCDE, 2019a, 2019b, 2020). Es un tránsito utópico porque añora el derecho, sin distinción, para acceder a las oportunidades digitales, para que niñas y niños desarrollen los saberes necesarios y para hacer del planeta un lugar, además de equitativo, sostenible (Unesco, 2015, 2019; Unicef, 2017). Sin embargo, el panorama descrito requiere, antes que euforia, un desapasionado alto en el camino para revisar dos implicaciones actuales en contra de la potencial oportunidad del aprendizaje digital.

Williamson (2018) describe este movimiento global como la cultura del hacer y el codificar. Niñas y niños aprendices de analistas de datos en procura de la soberanía de sí mismos para resolver con destreza digital las contradicciones y problemas sociales. Así, bajo este matiz de reconocida neutralidad, se edifica el comportamiento del ciudadano digital ideal (WiIliamson, 2016). En la misma dirección, Selwyn (2016) critica la concep- 
ción hegemónica de educación y tecnología digital que se construye sobre el ideal del aprendiz universal: alguien necesariamente motivado, con recursos disponibles, sociable por naturaleza, abnegado, abierto a experimentar, fallar con confianza y aprender a lo largo de la vida. En consecuencia, hay poca consideración con las diferencias que encierran la vida de los aprendices más desfavorecidos, se desestima la posibilidad del otro y las reprochables condiciones en las que viven seres humanos constreñidos, que con su mirada controvierten la romántica figura del aprendiz y el potencial ciudadano digital.

Aquí ubicamos el punto de inflexión que nos hace pensar en el aprendizaje digital de nuestras niñas y nuestros niños en situación de segregación escolar socioeconómica: ¿qué nos Ileva a pensar que la segregación escolar socioeconómica de niñas y niños limita la promesa de la potencia digital?, y ¿por qué es necesario visibilizarlos en este homogéneo marco del aprendizaje digital? Revisemos qué investigaciones hay al respecto.

Al referirnos a la segregación escolar, consideramos la distribución desigual de niñas y niños en las escuelas según rasgos personales o sociales (Dupriez, 2010; Ireson y Hallam, 2001). Para esta definición, Murillo (2016) reconoce tres manifestaciones: la segregación socioeconómica, la segregación étnico-cultural y la segregación por capacidades. Para nuestro problema particular acentuamos en la primera, resultado de sociedades, en particular las latinoamericanas, que por su disposición inequitativa favorecen la segmentación de niñas y niños en escuelas para ricos y escuelas para pobres (Murillo, 2019).

Las causas del fenómeno segregador son atribuibles a factores exógenos al sistema educativo, resultado de la histórica distribución desigual de los beneficios. Nada nuevo en nuestras latitudes que, de acuerdo con cifras de la calculadora de la desigualdad OXFAM (2015), el 71\% de la riqueza de América Latina y el Caribe está en manos del 10\% de la población. Además, las causas obedecen a factores endógenos, es decir, son producto de las decisiones de la administración del servicio educativo (Bellei, 2013; Krüger, 2019). Ahora bien, el debate no termina con ponderar la influencia de una u otra causa sobre el problema, todo lo contrario, apenas comienza, consiste en advertir que ambas son reversibles, porque su erradicación depende de voluntades humanas solo activadas al tiempo que nos negamos cómplices de cualquier amago de naturalización.

Entre todo, habría lugar para justificar la segregación bajo la tesis de la libertad de agrupación, la libre elección de escuela o la destinación de programas educativos focalizados para algunos sectores sociales más privilegiados. Sin embargo, estudios disponibles demuestran la intención de diferenciación social -elitización- que procuran algunos sectores sociales 
en desmedro de otros (Córdoba et al., 2020; Cortázar y Taberner, 2020; Murillo y Carrillo, 2020; Murillo y Graña, 2020a). Otras justificaciones posibles como el incentivo del mercado educativo (Murillo, 2016; Murillo y Martínez-Garrido, 2017b) hacen de la segregación escolar el producto de un juego de infra y sobreoferta escolar con consecuencias en la polarización social (Bonal y Zancajo, 2020).

A la reversibilidad de la segregación escolar sumemos las consecuencias como una razón adicional para pensar su desnaturalización. Permitir sistemas educativos segmentados disminuye la posibilidad del encuentro de niñas y niños de diferentes orígenes socioeconómicos, por tanto, la labor escolar de cohesión social, encuentro y socialización está comprometida (Murillo y Duk, 2016). Por una parte, los más desfavorecidos pierden la oportunidad del reconocido efecto pares, beneficioso en los rendimientos escolares (Krüger, 2020; Murillo et al., 2018; OCDE, 2012, 2013, 2016), además, experimentan un impacto negativo en las expectativas de aprendizaje, el autoconcepto y la confianza (Murillo y Duk, 2016). De acuerdo con Krüger (2019), la situación de segregación escolar se constituye en un doble riesgo: además de la evidente dificultad que reviste el origen social desfavorecido para el éxito educativo, la alta probabilidad de asistir a escuelas con estudiantes en similares condiciones aporta un punto en contra a los logros educativos, profundiza la desigualdad en los aprendizajes y lastima las potenciales oportunidades laborales futuras. En la contraparte, los estudiantes de mayores recursos ven reducidas las posibilidades de educarse en escenarios incluyentes y diversos, por tanto, están limitados a versiones parciales y homogéneas de realidad que alimentan el discurso del nosotros/ otros, los deseados/indeseados (Córdoba et al., 2020).

No estamos ni mucho menos frente a una problemática tímida e incipiente. El fenómeno segregador campea por las sociedades sin distinción, a tal magnitud y evolución, que es capaz de limitar los aprendizajes y dejar a la retaguardia las esperanzas de un futuro digitalizado (Bonal, 2018; Charmillot y Felouzis, 2020; Ferrando et al., 2020; Lisboa et al., 2020; Martínez-Garrido et al., 2020; Murillo y Martínez-Garrido, 2017a; Ramírez y Vazquez, 2020). Apostamos por hacer conciencia en este apartado de la limitación para, desde nuestro rol de profesores, abrazar acciones para que niñas y niños no sigan viendo, en palabras de Galtung (1969), comprometido su potencial nivel de realización educativa.

Por tanto, nos preguntamos: ¿cómo estimular aprendizajes digitales a pesar de la situación de segregación escolar socioeconómica?, al definirlo, ¿qué aprendizajes logran estimular niños y niñas? En este caso, apelamos a la investigación acción, o al concurso del profesor-investigador y sus estu- 
diantes, para focalizar una limitación, desarrollar un plan para hacer frente a la limitación y recuperar, analizar e interpretar los aprendizajes digitales construidos. Por tanto, proponemos dos objetivos:

a) Ensayar una estrategia educativa en pensamiento computacional, como catalizadora, para construir aprendizajes digitales junto a niñas y niños en situación de segregación escolar socioeconómica.

b) Valorar e interpretar los aprendizajes digitales construidos.

\section{Método, o hacer frente a la limitación}

El estudio correspondió a una investigación acción práctica (Creswell, 2012; Schmuck, 2006) construida durante un año escolar entre el docenteinvestigador y sus estudiantes de escuela primaria. Empleamos, de acuerdo con la taxonomía de Mills (2018), técnicas de indagación y examinación para la recolección de datos. Para la gestión y procesamiento de los datos usamos el software de análisis cualitativo Atlas ti versión 8. En particular, usamos el modelo para el análisis de datos asistido por computadora NCT -Noticing, Collecting and Thinking- (Friese, 2019).

\subsection{El contexto y los participantes del estudio}

El desarrollo investigativo procedió en la institución educativa pública "La Camila" que presta servicios de educación preescolar, básica primaria y secundaria. La institución está emplazada en el municipio de Bello, Colombia. Alberga estudiantes con nivel socioeconómico (NSE) 1 y 2 (los niveles más bajos de una escala de 1 a 4), de acuerdo con los datos históricos 20152019 arrojados por las pruebas nacionales Saber ICFES. Concentra, además, solo estudiantes habitantes del mismo barrio. En consecuencia, los niños y las niñas cuentan con altas probabilidades de encontrarse y conducir sus experiencias de escolares con pares en la misma situación socioeconómica. Fenómeno segregador, descrito anteriormente, con graves consecuencias para el aprendizaje.

Los participantes del estudio un profesor-investigador, 71 estudiantes -33 niñas y 38 niños entre los 9 y 13 años de edad-y sus tutores, que con consentimiento aceptaron desarrollar la estrategia de pensamiento computacional. 
Figura 1. Infografía caracterización socioeconómica de los participantes del estudio.

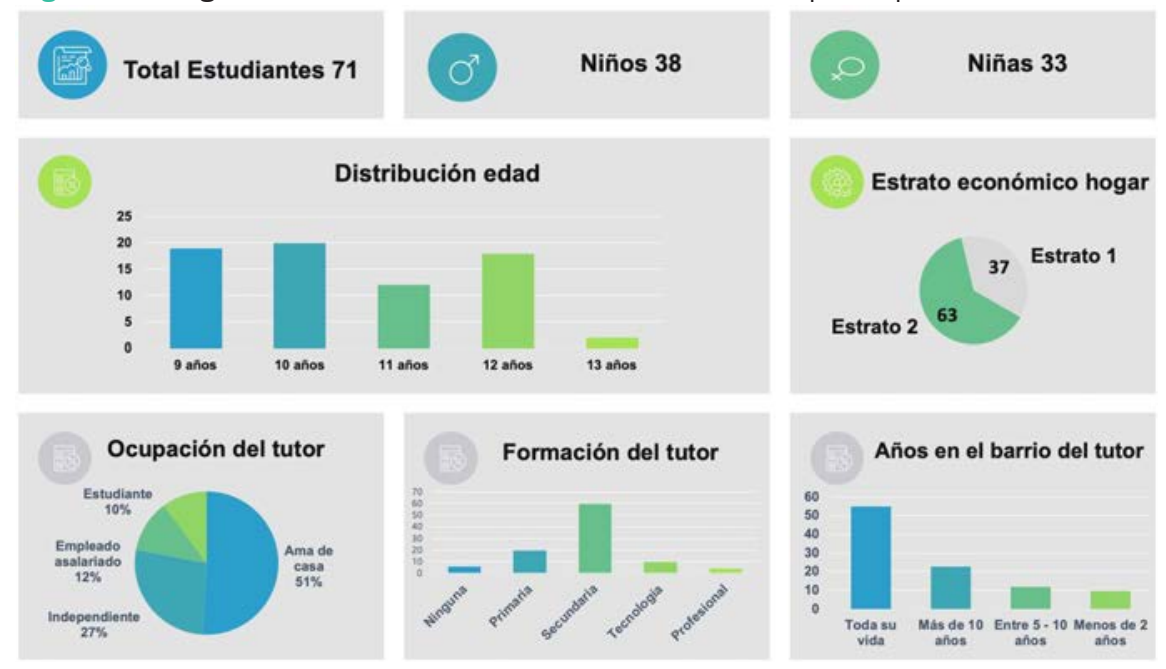

Fuente: elaboración propia.

\subsection{Procedimiento}

\subsubsection{La estrategia de pensamiento computacional}

En el marco de las políticas públicas colombianas CONPES 3975 y 3988, la formación de aprendizajes dirigidos a la transformación digital y la sociedad de la revolución 4.0 son imperativos para habilitar condiciones hacia una sociedad equitativa. Por tanto, la estrategia de pensamiento computacional IUNGO, presentada en el banco de contenidos del Ministerio de Educación Nacional, fue adoptada como mediador pedagógico para catalizar aprendizajes digitales de niñas y niños en situación de segregación escolar socioeconómica. Anticipamos que, si bien, las condiciones sociales y ecónomicas desfavorables hacen difícil la labor educativa, no menos cierto es que la esperanza de las comunidades vulnerables está en el colectivo de docentes y directivos capaces de promover acciones adaptadas a las necesidades de la situación escolar.

Por tanto, niñas y niños desarrollaron 7 módulos en línea y desconectados, que consistían en resolver problemas a través de juegos que implican el desarrollo de habilidades de programación por bloques, de acuerdo con la guía didáctica provista por la estrategia IUNGO Education, secuenciación, depuración, acciones, parámetro variable, ciclos y parámetro variable con ciclos. 
Para iniciar el proceso, las niñas y niños participaron de un taller de inducción a la estrategia de pensamiento computacional, para aprender a acceder a la plataforma digital e identificar el contenido de las actividades. Llevaron a sus casas una comunicación escrita para informar del desarrollo de la actividad y solicitar el consentimiento a sus tutores, para poder participar; de vuelta, recibimos 71 autorizaciones firmadas por los tutores de niñas y niños.

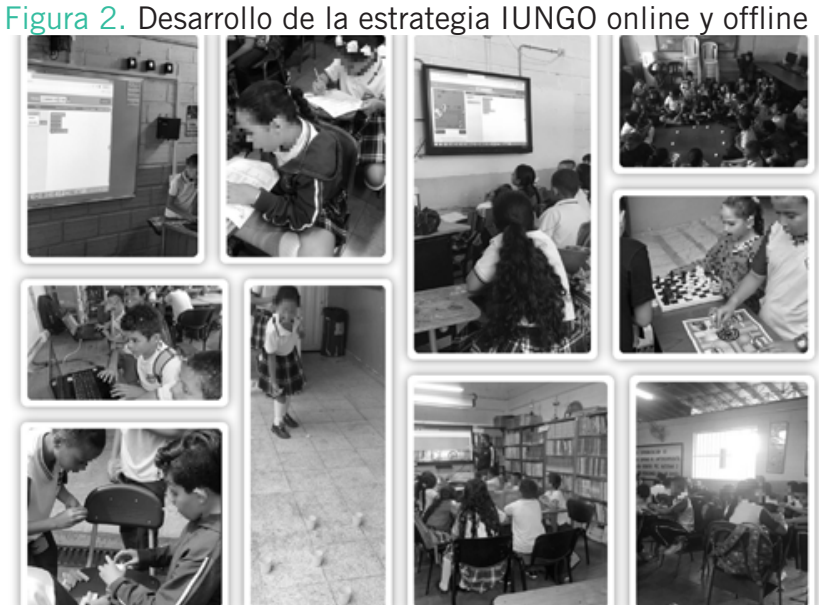

Fuente: elaboración propia.

\subsubsection{Administración de grupos focales}

El avance exitoso en la plataforma de 10 niñas y 11 niños, además del elevado interés, que se manifestó en sus inquietudes y en las conversaciones extraclase, devino en la pregunta por la experiencia que vivían, más allá de los resultados arrojados por el aplicativo web. Entonces, realizamos dos sesiones de grupos focales. La primera integrada por 4 niñas y 9 niños y la segunda por 6 niñas y 2 niños.

Figura 3. Extracto de construcciones de niños y niñas en grupos focales

Fuente: elaboración propia.

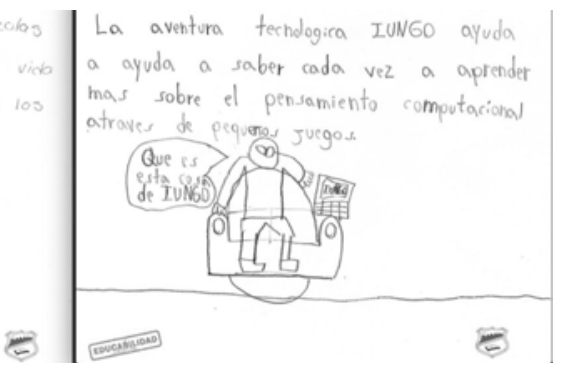




\subsubsection{Desarrollo de talleres de clase}

Juicio al Internet. Ambientamos el espacio del salón de informática como un juicio inquisidor medieval, porque las familias denuncian los daños nocivos que las tecnologías digitales hacen a las niñas y los niños. Los estudiantes podían posicionarse a favor o en contra, conversaban y presentaban un alegato para absolver o defenderlas.

Figura 4. Taller juicio al Internet.

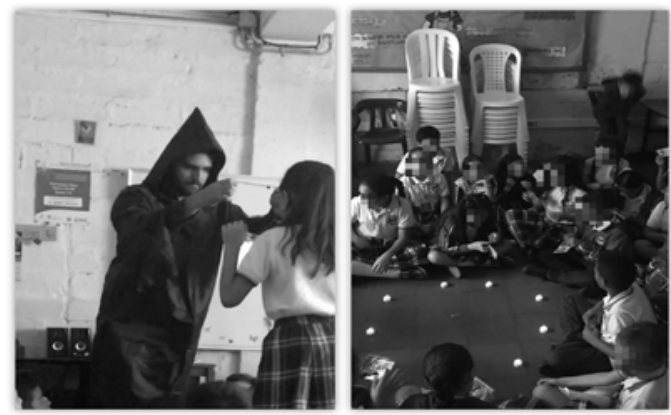

Fuente: elaboración propia.

Cuento sobre Internet. La consigna: un personaje real o ficticio, que podrían ser ellos mismos, luego relatar una historia en la cual el mundo del Internet fuera protagonista. Los relatos nutrieron el inventario de saberes y perspectivas que los estudiantes ofrecen a su relación con la Internet.

Figura 5. Taller cuento sobre Internet

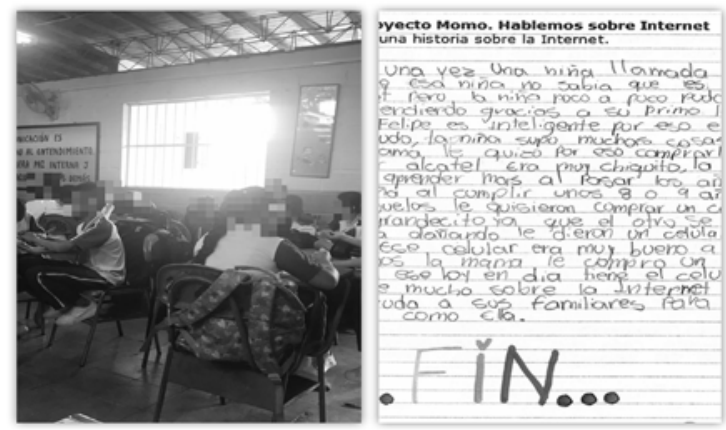

Fuente: elaboración propia

Árbol de habilidades en Internet. Los estudiantes tenían al frente un árbol de otoño: hojas caídas que representaban las habilidades de los abuelos y los padres. Tenían la responsabilidad de reflorecer el árbol, de brindar un follaje nutrido de hojas que al respaldo llevaran inscritas las mayores habilidades que se poseen al estar en Internet 
Figura 6. Taller árbol de habilidades digitales
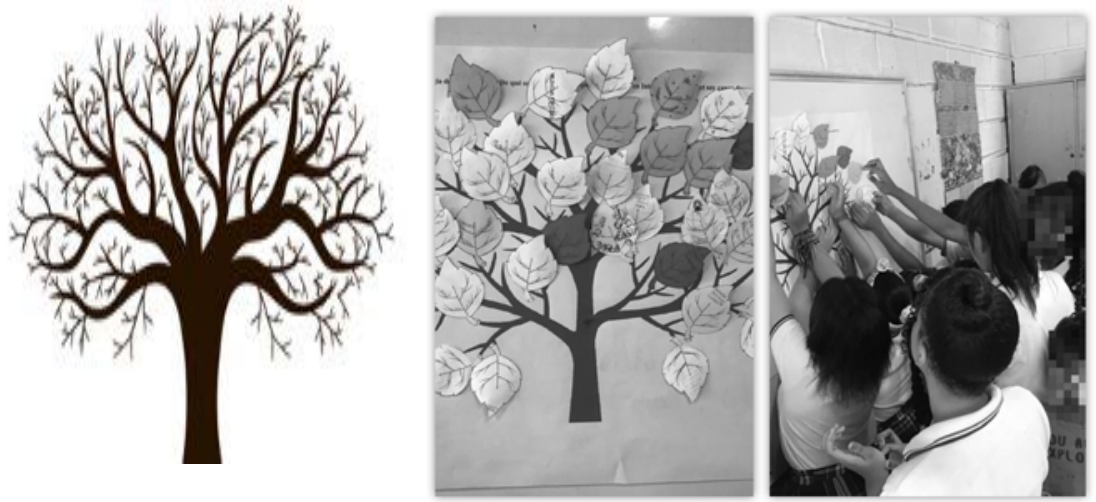

Fuente: elaboración propia.

Graduación, o la recuperación familiar de la experiencia vivida. Queríamos, además de resaltar el loable esfuerzo de estudiantes y tutores, razón suficiente para el evento de graduación de la estrategia digital, reconstruir la experiencia vivida de semanas de dedicación a través de una actividad formativa. Los estudiantes y sus tutores recrearon una sesión en vivo de un algoritmo que los Ilevaría a una aula de clase, ambientada con imágenes y memorias del trabajo con la plataforma; donde quisimos evocar y logramos relatos con alto valor empírico.

Figura 7. Graduación estrategia IUNGO
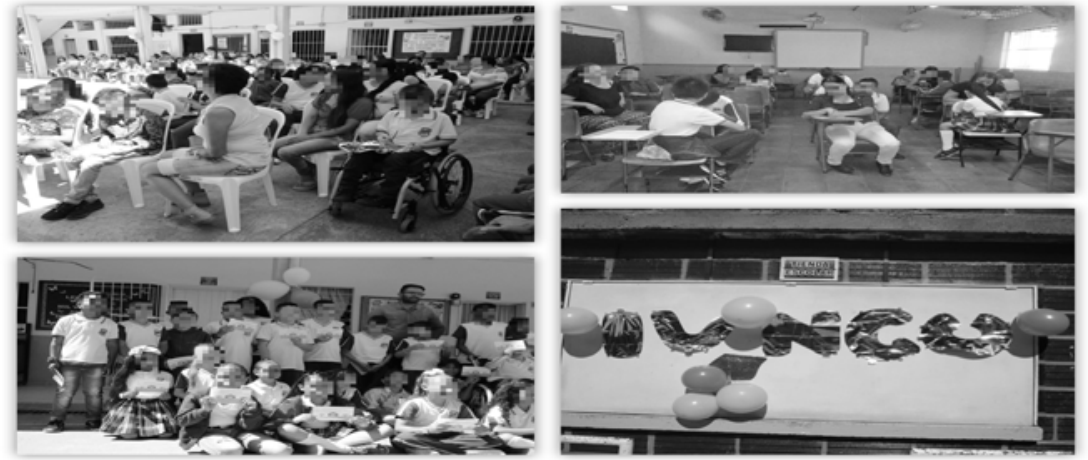

Fuente: elaboración propia.

\subsection{Análisis de los datos}

Acudimos a la propuesta de Friese (2019) para el análisis iterativo y recursivo de datos cualitativos a través del software Atlas ti. Organizamos los documentos de texto recuperados de los grupos focales, talleres en clase y experiencia familiar vivida. Los documentos fueron nomenclados con 
el participante y tipo de técnica empleada. La identificación y agrupación permitió evaluar la validez interna de los análisis basados en la coocurrencia de los datos entre las distintas técnicas y los participantes consultados como se datallará más adelante.

Paso seguido, ofrecimos una lectura a cada documento y procedimos a la codificación inicial (Charmaz, 2006). En esta primera codificación etiquetamos 200 segmentos de textos, usamos 45 códigos. Este paso nos ofreció una visión general del contenido de los datos. Luego, clasificamos los códigos para superar el fango de códigos o swamp code generado (Friese, 2019). A través de la función de filtro del sotfware, redujimos a 8 la lista de códigos bajo el principio de ganar mayor nivel de abstracción, es decir, al fusionar códigos descriptivos que entrañaban relación. En esta fase iniciamos la escritura de memos analíticos tempranos que describían el contenido de los códigos.

A continuación, iteramos hacia los datos. Con la función de filtro realizamos una nueva codificación, ahora focalizada (Charmaz, 2006). Así evaluamos la pertinencia de citas textuales dentro de los códigos, corregimos redundancia de citas y mutua exclusividad entre estos (Friese, 2019). En este punto, alcazamos el nivel de saturación de los códigos. Procedimos a escribir memos analíticos, que constituyeron el germen de los resultados del estudio. Interpretamos y elaboramos argumentos fundamentados en citas textuales ejemplares (Charmaz, 2006).

Finalmente, aplicamos dos indicadores de validez interna al estudio. Primero, el índice de co-ocurrencia código-documento y, segundo, el índice de representatividad código-participante. Ambos conocidos en la literatura como triangulación, o el proceso de corroborar la evidencia desde diferentes métodos de recolección de información y desde diferentes individuos participantes.

En la tabla 1, el indicador de co-ocurrencia permite establecer la recurrencia absoluta y relativa de los códigos (aprendizajes digitales) en cada uno de los grupos de documentos analizados (métodos de investigación). Este indicador es útil para validar que la construcción de los resultados del estudio cuente con la riqueza de diferentes fuentes documentales de indagación y examinación, no de un unívoco y reducido instrumento de investigación. En otras, la triangulación por métodos de recolección de información (Creswell, 2012). 
Tabla 1. Indicador de co-ocurrencia código-documento

\begin{tabular}{|c|c|c|c|c|c|c|c|}
\hline \multirow[t]{2}{*}{ Códigos } & \multicolumn{7}{|c|}{ Grupo documento } \\
\hline & Diario & Árbol & Cuento & $\begin{array}{l}\text { Gradua- } \\
\text { ción }\end{array}$ & $\begin{array}{l}\text { Grupo } \\
\text { focal }\end{array}$ & $\begin{array}{l}\text { Jui- } \\
\text { cio }\end{array}$ & $\begin{array}{c}\text { Tota- } \\
\text { les }\end{array}$ \\
\hline Aprendizajes espontáneos & $\begin{array}{l}27,7^{*} \\
(5)^{* *}\end{array}$ & - & - & $\begin{array}{l}72,2 \\
(13)\end{array}$ & - & - & $\begin{array}{l}100 \\
(18)\end{array}$ \\
\hline Censura de contenidos & - & - & $\begin{array}{l}40 \\
(2)\end{array}$ & $\begin{array}{l}20 \\
(1)\end{array}$ & - & $\begin{array}{l}40 \\
(2)\end{array}$ & $\begin{array}{l}100 \\
(5)\end{array}$ \\
\hline Enseñanza a la familia & - & - & $\begin{array}{c}33,3 \\
(2)\end{array}$ & - & $\begin{array}{c}16,6 \\
(1)\end{array}$ & $\begin{array}{l}50 \\
(3)\end{array}$ & $\begin{array}{l}100 \\
(6)\end{array}$ \\
\hline $\begin{array}{l}\text { Identificación de los propios apren- } \\
\text { dizajes }\end{array}$ & - & - & $\begin{array}{c}12 \\
(12)\end{array}$ & $\begin{array}{c}47 \\
(47)\end{array}$ & $\begin{array}{l}38 \\
(38)\end{array}$ & $\begin{array}{l}3 \\
(3)\end{array}$ & $\begin{array}{c}100 \\
(100)\end{array}$ \\
\hline $\begin{array}{l}\text { Pertenencia a grupos de interés en } \\
\text { línea }\end{array}$ & - & $\begin{array}{c}52 \\
(13)\end{array}$ & $\begin{array}{l}20 \\
(5)\end{array}$ & - & - & $\begin{array}{l}28 \\
(7)\end{array}$ & $\begin{array}{l}100 \\
(25)\end{array}$ \\
\hline Habilidad para jugar en línea & $\begin{array}{c}11,1 \\
(1)\end{array}$ & $\begin{array}{l}55,5 \\
(5)\end{array}$ & - & - & - & $\begin{array}{c}33,3 \\
(3)\end{array}$ & $\begin{array}{l}100 \\
(9)\end{array}$ \\
\hline Sentimientos frente al aprendizaje & - & - & $\begin{array}{l}58,6 \\
(17)\end{array}$ & - & $\begin{array}{l}41,3 \\
(12)\end{array}$ & - & $\begin{array}{l}100 \\
(29)\end{array}$ \\
\hline Utilidad escolar de la tecnología & - & $\begin{array}{c}37,5 \\
(3)\end{array}$ & $\begin{array}{l}12,5 \\
(1)\end{array}$ & - & - & $\begin{array}{l}50 \\
(4)\end{array}$ & $\begin{array}{l}100 \\
(8)\end{array}$ \\
\hline Totales & $\begin{array}{c}3 \\
(6)\end{array}$ & $\begin{array}{l}10,5 \\
(21)\end{array}$ & $\begin{array}{l}19,5 \\
(39)\end{array}$ & $\begin{array}{l}30,5 \\
(61)\end{array}$ & $\begin{array}{l}25,5 \\
(51)\end{array}$ & $\begin{array}{c}11 \\
(22)\end{array}$ & $\begin{array}{l}100 \\
(200)\end{array}$ \\
\hline
\end{tabular}

Fuente: elaboración propia.

Nota: *valor relativo \% de citas textuales asociadas a cada grupo de documentos y etiquetadas bajo el código. **valor absoluto \# de citas textuales asociadas en cada grupo de documentos y etiquetadas bajo el código.

Tabla 2. Indicador de representatividad código-participante

\begin{tabular}{|c|c|c|c|c|c|}
\hline \multirow[t]{2}{*}{ Códigos } & \multicolumn{5}{|c|}{ Participante } \\
\hline & @niña & @niño & @familia & $\begin{array}{l}\text { @profesor- } \\
\text { investigador }\end{array}$ & Total citas \\
\hline Aprendizajes espontáneos & - & - & $\begin{array}{l}72,2 \\
(13)\end{array}$ & $\begin{array}{l}27,7 \\
(5)\end{array}$ & $\begin{array}{l}100 \\
(18)\end{array}$ \\
\hline Censura de contenidos & $\begin{array}{l}60^{*} \\
(3)^{* *}\end{array}$ & $\begin{array}{l}20 \\
(1)\end{array}$ & $\begin{array}{l}20 \\
(1)\end{array}$ & - & $\begin{array}{l}100 \\
(5)\end{array}$ \\
\hline Enseñanza a la familia & $\begin{array}{c}66,6 \\
(4)\end{array}$ & $\begin{array}{c}33,4 \\
(2)\end{array}$ & - & - & $\begin{array}{l}100 \\
(6)\end{array}$ \\
\hline $\begin{array}{l}\text { Identificación de los propios } \\
\text { aprendizajes }\end{array}$ & $\begin{array}{l}36 \\
(36)\end{array}$ & $\begin{array}{l}17 \\
(17)\end{array}$ & $\begin{array}{c}47 \\
(47)\end{array}$ & - & $\begin{array}{l}100 \\
(100)\end{array}$ \\
\hline $\begin{array}{l}\text { Pertenencia a grupos de interés } \\
\text { en línea }\end{array}$ & $\begin{array}{l}16 \\
(4)\end{array}$ & $\begin{array}{c}84 \\
(21)\end{array}$ & - & - & $\begin{array}{l}100 \\
(25)\end{array}$ \\
\hline Habilidad para jugar en línea & $\begin{array}{c}22,2 \\
(2)\end{array}$ & $\begin{array}{l}66,6 \\
(6)\end{array}$ & - & $\begin{array}{c}11,1 \\
(1)\end{array}$ & $\begin{array}{l}100 \\
(9)\end{array}$ \\
\hline Sentimientos frente al aprendizaje & $\begin{array}{l}37,9 \\
(11)\end{array}$ & $\begin{array}{l}34,4 \\
(10)\end{array}$ & $\begin{array}{l}27,5 \\
(8)\end{array}$ & - & $\begin{array}{l}100 \\
(29)\end{array}$ \\
\hline Utilidad escolar de la tecnología & $\begin{array}{c}37,5 \\
(3)\end{array}$ & $\begin{array}{c}12,5 \\
(1)\end{array}$ & $\begin{array}{l}50 \\
(4)\end{array}$ & - & $\begin{array}{l}100 \\
(8)\end{array}$ \\
\hline Total participación & $\begin{array}{l}31,5 \\
(63)\end{array}$ & $\begin{array}{l}29 \\
(58)\end{array}$ & $\begin{array}{l}36,5 \\
(73)\end{array}$ & $\begin{array}{c}3 \\
(6)\end{array}$ & $\begin{array}{l}100 \\
(200)\end{array}$ \\
\hline
\end{tabular}

Fuente: elaboración propia.

Nota: *valor relativo \% de citas textuales asociadas a cada participante del estudio y etiquetadas bajo código. **valor absoluto \# de citas textuales asociadas a cada participante del estudio y etiquetadas bajo código. 
En la tabla 2, por su parte, el indicador de representatividad permite establecer la recurrencia absoluta y relativa de los códigos (aprendizajes digitales) en las intervenciones de cada uno de los participantes de la investigación-acción. Este indicador es útil para garantizar un equilibrio en la participación y contraste de versiones de realidad. En primer lugar, permitió que tanto la voz del niño y de la niña tuvieran la misma relevancia (peso) en la construcción de los aprendizajes. En segundo lugar, permitió una construcción de aprendizajes contrastada por las intervenciones de la familia y el profesor-investigador. En otras, la triangulación de participantes (Denzin y Lincoln, 2005).

\section{Resultados, o revelar saberes digitales construidos \\ 4.1 Aprendizajes espontáneos}

La estrategia digital generó un haz de aprendizajes espontáneos que para las familias significó un espectro entre preocupación, reconocimiento y despreocupación. La preocupación percibe a las niñas y niños como aprendices aventajados de la tecnología digital que deben ser orientados, restringidos, vigilados o persuadidos para que hagan usos productivos y responsables. La mayor preocupación significa la crisis del aprendizaje escolar tradicional: hacer las tareas, responder de memoria, hacer las transcripciones o no quedarse atrasado en los cuadernos. Aún así, hay un reconocimiento a la propuesta digital porque capta la atención, eleva la motivación y desarrolla habilidades que ni la familia, ni la escuela podrían desarrollar. Sin embargo, es un tímido reconocimiento que transita entre la impavidez para dar respuesta a estos inusitados aprendizajes y la sensación de pérdida que resigna la obligatoria mediación adulta para el aprendizaje infantil.

Los niños solos de forma espontánea han aprendido a manejar las herramientas digitales. Nunca como padres hemos orientado ningun aprendizaje. Aunque unos les explica cuando están pequeños cómo se manejan los dispositivos, pero de forma muy elemental (@familia_graduación, 2:29). ¿Cómo hace esas cosas? No sé, porque la verdad yo no le dedico tiempo para aprender todo eso, lo único es que me pide en ocasiones el celular para realizar alguna actividad. Eso la verdad me sorprende porque no es necesario que haya un adulto al frente y que solos sean capaces de aprender a usar toda la tecnología (@familia_graduación, 4:26).

En el otro costado, la despreocupación hace presencia como justificación para dejar solos a las niñas y los niños frente a la pantalla porque los tutores no tienen mucho que enseñar o porque el saber de niñas y niños rebasa las respuestas que pueden aportar los tutores, esto deriva en asumir 
que la tecnología por sí misma puede enseñar. Esto se ratifica en las narrativas de los niños y niñas que admiten haber aprendido solos y que los tutores no les enseñaron nada de lo que logran conocer.

La tecnología para la educación de mi hijo me parece muy buena porque yo no tengo que estar muy preocupada de estar muy pendiente de consultas o trabajos porque con la tecnología puede aprender a leer a sumar, muchas cosas que uno no tiene tiempo de enseñarle, pero que él se sienta ahí y las aprende ahí mismo (@familia_graduación, 14:15).

Yo aprendí a usar Internet sola, nadie me ha ayudado (@niña_árbol, 24:15).

Por su parte, el profesor-investigador valora en su diario la capacidad de libre asociación, cooperación y búsqueda colaborativa de las soluciones a los retos más difíciles de la estrategia digital. Exhalta el ejemplo de la biblioteca escolar recreada que, pasó de ser un espacio de consulta estática, individual y silenciosa, al espacio del movimiento del cuerpo como experimentación y el encuentro recíproco entre niñas y niños. Afirma el profesor-investigador la capacidad de los estudiantes para justificar, ante el escepticismo del tutor, la importancia de lo que aprenden. Por último, resalta la transición del estadio de la desmotivación, el desgano y el pasar desapercibido en el que viven niños y niñas en la escuela tradicional, a la actitud de interés, ganas de superar retos y figurar ante sus compañeros como quien procuró la estrategia digital.

Afirma un niño en biblioteca: Este fin de semana me dediqué a terminar los niveles de lungo. Descubrí que avanzando en retos de la misma semana puedo entender el que no logré hacer antes porque los de la misma semana tienen características muy similares. El mismo juego me enseña cuando tengo dificultades o dudas (@profesor-investigador_diario, 428:56)

\subsection{Censura de contenidos}

La censura de contenidos es un saber que las niñas y los niños evidencian en sus relatos: el rechazo a manifestaciones inadecuadas en sus experiencias digitales. La censura tiene un cariz de recomendación y exhortación a los demás niños y niñas para que eviten los contenidos ofensivos o las amenazas del desconocido que está al otro lado de la pantalla.

Las personas no deberían ver porno (@niña_juicio, 24:21).

Alguna vez le dije a un amigo que no sabía que no se metiera a páginas groseras porque eso no estaba bien hecho. No viera cosas malas, solo vídeos así de risa no más (@niño_árbol, 25:20).

La censura implica no hacer lo mismo que hacen sus pares porque es moda o tendencia. 
A mis compañeros les gusta hablar con gente más grande y desconocidos son ellos pero yo no. He aprendido que no podemos meternos en Facebook a hablar con gente que no conocemos porque nos pueden hacer daño (@ niña_cuento, 43:21).

La censura no es una respuesta temerosa, también invita a reaccionar ante lo atroz en los contenidos digitales. Destacamos la capacidad de niñas y niños para elaborar itinerarios de autocuidado digital.

En Internet no está bien hecho amenazar a otros o montar vídeos sobre las otras personas. Cuando veo una cosa desagradable en Internet yo me salgo, si me meto y vuelve a salir yo llamo y le muestro a mi mamá. En Internet no está bien hecho ver cosas feas, con malas palabras y groseras (@niña_juicio, 40:16).

La censura relaciona la intimidad como un bien preciado que no debería sobrepasarse porque ninguna persona debería ser expuesta en las redes sin su consentimiento. La censura implica depurar la lista de amigos y establecer contacto solo con personas conocidas. Censurar implica aprender de las experiencias de otros representados en otros medios de comunicación. La censura permite reconocer que Internet no es malo, sino que la maldad proviene de los usos indebidos que hacen las demás personas.

No me pasó a mi, pero yo veo la Rosa de Guadalupe. Una vez una niña se tiró de un puente y casi que se moría porque le decían cerda. Le tomaban una foto como en una fiesta con todos y a ella le pusieron una cara de marrano y montaron todas las fotos a Internet y se la empezaron a gozar (@niña_cuento, 31,6).

(...) un día Sofía no se quería ni mover de la cama, Iloraba inconsolablemente, ni decia una sola palabra. La mamá la llamaba: "Sofía, Sofía", ni le respondía. Por la tarde, Sofía estaba dormida y su madre le cogió el celular... leyó y leyó y su madre comenzó a llorar... la habían acosado sexualmente en su Facebook. Ella le dijo todo lo que había pasado, ya se arreglaron las cosas y jamás volvió a mirar las redes sociales (@niña_cuento, 354:1).

Por último, la censura es la defensa que la niña implora por saberse víctima del inminente riesgo que atraviesa su cuerpo en Internet. Es evidente la desigualdad de género expresada en una experiencia digital menos afortunada y sentida como riesgo para las niñas. Por su parte, en los relatos, la experiencia de los niños es más favorable en cuanto a espacio de experimentación, libertad y aprendizaje. La experiencia digital de las niñas antes que posibilidad, como en el caso de los niños, denota una limitación del potencial digital. 


\subsection{Enseñanza a la familia}

Las niñas y los niños en casa apoyan a sus tutores en labores cotidianas que implican el uso de tecnologías digitales. Los tutores admiten que son superados en habilidades y que aprenden de sus tutoriados. Encontramos tutores que aún no saben leer y escribir, por tanto, la ayuda de sus hijos e hijas escolarizados es definitiva para atender diligencias que obligan hoy por hoy a acceder a Internet. Las niñas y los niños disfrutan enseñando a sus familiares a través de un intercambio de roles que invierte la labor de orientación y los consejos, ahora desde éstos hacia sus padres.

Juanita dígamos de un $100 \%$ ha aprendido en internet un $80 \%$ porque ella a veces, le he tenido que decir: "hija ayúdame con esto para buscarlo que no lo encuentro" o si estoy muy ocupada le digo: "Juanita" ayúdame a buscar esto para mi trabajo, tengo que responder esto en Internet, mirá ayudámelo a buscar. Es una niña muy capacitada para usar internet (@familia_graduación, 15:25). Ayudo a mi mamá para que se meta en Internet (@niño_árbol, 18:14).

Además, la labor de enseñar exige la persuasión para que sus tutores valoren el uso de tecnologías digitales. Implica persuadir en que si bien hay peligros, también pueden ser advertidos y con su acompañamiento evadidos.

(...) una niña Ilamada Sara que le encantaba Internet con su papá y mamá a la abuela de Sara no le gustaba el Internet dice que parece peligroso. Sara le insistió que no era peligroso antes era divertido, pero su abuela no le hizo caso (...) A la abuela de Sara su mamá le compró un celular para que comprobara que no era aburrido, ni peligroso. Sara le enseñó a manejar el celular a la abuela. Desde ese día la abuela también pasaba alegre y toda la familia alegre con internet (@niña_cuento, 339:1).

\subsection{Identificación de los propios aprendizajes}

Niñas y niños identifican como los mayores aprendizajes: el ensayo y error; la solución de problemas; la apropiación de la jerga, propia de la programación computacional; y, la relación del contenido con la vida diaria.

Aprendimos a generar algoritmos, a resolver problemas lógicos, a pensar en procesos que nos permiten llegar a un objetivo, a generar diferentes alternativas o caminos para llegar al fin (@niño_focal, 49:2).

Aprendí que con lungo uno puede decidir qué camino tomar como un algoritmo y seguir su sueño y poder conseguir lo que queremos como los personajes (@niña_focal, 52:2).

A hacer algoritmos, poner comandos, a mejorar mi memoria con los juegos entretenidos. A seguir órdenes lógicas, ordenadas y estructuradas (@ familia_graduación, 47:2) 
Al fin, valorar la importancia escolar del orden, la memoria o la concentración que antes no podía ser comprendida a través del regaño en clase o la imposición de la norma. A relacionar las tareas escolares con el pensamiento organizado, planeado como medios para lograr una meta. A relacionar o integrar los saberes aprendidos con nociones de las asignaturas de la escuela.

Concentración, agilidad en cada proceso. Disciplina para completar cada semana. Interesante para mis aprendizajes como alumno (@niña_focal, 73:2).

Podemos compararlo con los ejercicios que hacemos en la clase de Educación Física. Debemos seguir unas instrucciones, un orden para hacer bien los ejercicios (@niño_focal, 404:23)

\subsection{Pertenencia a grupos de interés}

La relación entre pares del colegio puede extenderse a escenarios digitales que procuran conflicto y negociación dentro de la jornada escolar. El juego de FreeFire coincidió con el proceso de trabajo de campo. Esta tendencia implicaba las asociaciones entre pares, las discusiones en torno al juego, las apuestas y retos, conflictos escolares y la desviación de los contenidos escolares, al sentido y la perspectiva del juego que estaba de moda.

Yo a través de Internet he hablado con personas desconocidas a través del juego en línea de FreeFire, perosnas de México y Chile (@niño_árbol, 21:13). Mis compañeros prefieren el juego de FreeFire, ahora estamos muy conectados todos con ese juego (@niño_árbol, 41:20).

\subsection{Habilidad para jugar en línea}

El juego es atractivo por la adicción que produce, el deseo de alcanzar los niveles, superar a los demás y conformar grupos de interés escolar donde se miden capacidades y se permite la competencia para determinar quién es el más habilidoso. El juego se convierte en el tema central de la asistencia a la escuela. La estructura escolar entra en contradicción entre el saber que debería enseñar y el saber que en realidad circula por los pasillos y en las expectativas de los estudiantes.

A todos los niños les gusta FreeFire es adictivo, trata de disparos, uno tiene que ir mejorando, comprando personajes e ir ganando partidas. Lo que más me gusta hacer es jugar (@niño_juicio, 19:16).

A mis compañeros les gustan los juegos, hablan todo el día sobre FreeFire (@niña_árbol, 19:23). 


\subsection{Sentimientos frente al aprendizaje}

El aprendizaje digital no es restingido a lo racional, calculador y con propósitos productivos. La ventana digital proyecta haces de sensibilidades y emociones. Estimula la empatía con los personajes que aparecen en pantalla. Las narraciones sobre el contenido digital son evocadoras del recuerdo.

El computador nos ha acompañado desde que tenía cuatro años, yo me tranqulizo mucho en ella. Además, mi primita pequeña escucha música y me da mucha risa como baila (@niña_juicio, 39:13).

Me gusta ver videos donde la gente es solidaria con otras personas que no tienen nada (@niño_árbol, 41:12).

Al terminar los deberes escolares, comienza el episodio más creativo porque se puede acceder a Youtube y se ocurren ideas geniales, increíbles y únicas. Por tanto, no tener acceso digital es "comparable con un inservible juguete sin bateria" (@niño_cuento, 330:19). Los episodios que significan restricción de acceso por no hacer las tareas o portarse mal son vividos con tristeza solo superada con el retorno al acceso. Así, la experiencia digital es de confort, se describe en la escena de estar en el cuarto tomando un chocolatico caliente.

YouTube me trae felicidad y a veces cuando termino mis tareas utilizo Internet a veces se ocurren ideas geniales, increíbles y únicas. Cuando termino las tareas me tiro en el sofá pongo música de YouTube y me tomo un chocolatito caliente (@niña_cuento, 330:12).

\subsection{La utilidad escolar de la tecnología digital}

Hay acuerdos y desacuerdos entre participantes. Hay acuerdo, porque se pueden aprender cosas que pueden servir para el futuro y para lograr las metas. La tecnología digital facilita mucho las tareas de la escuela y evita desplazarse lejos para hacer las consultas, ahorra tiempo.

El internet nos sirve para alcanzar nuestras metas y lograr nuestros sueños (@niña_juicio, 195:1).

Estoy de acuerdo con que Internet aprende más y más rápido desde que estén haciendo lo correcto... por ejemplo se reduce la cantidad de tiempo para tareas básicas como buscar información, eso es un beneficio para el estudiante ya que no tiene que salir de la casa para investigar sus tareas (@familia_graduación, 11:26).

Hay acuerdo en la importancia de la tecnología digital, porque el mundo cambia y la escuela debe procurar también al cambio: atender a las nuevas expectativas de las niñas y los niños. Sin embargo, la escuela no debe depender de la tecnología digital, debe procurar los límites para su uso. 
La escuela debe usar tecnología antes no era necesario, pero ahora la generación va cambiando por esto es necesario que la escuela esté actualizada para poder darle esa información a los niños usando esa tendencia que hay por ahí (@familia_graduación, 5:21).

Estas narrativas son matizadas entre tantas habilidades espontáneas, recreativas y ociosas, pero que encuentran poca relación con la productividad escolar.

Un sobrino perdió el año sexto, pero día y parte de la noche está ahí metido y hágale... en el celular o computador. Se dedica a todo menos a estudiar. Necesita algo que consultar en internet y ahí mismo la llamada para la tía o el tío o para consultar en el computador iporque no sabe cómo hacerlo! Yo le digo, dejá la bobada, coja y busque (@familia_graduación, 411:15).

Las habilidades pueden estar al servicio de la tarea escolar, pero requieren un enfoque docente, aprender a ponerlas al servicio de los aprendizajes escolares, de lo contrario, la tecnología es más conflictiva y problemática que aportante, porque dispersa la atención y roba la motivación.

Frente a las tecnologías siempre debe estar el adulto responsable quien se convertirá en el custodio del tiempo de la calidad y del contenido a seguir (@familias_graduación, 429:20).

\section{Discusión y conclusiones, 0 de reflexionar sobre lecciones aprendidas}

Ratificamos con los aprendizajes espontáneos y la utilidad escolar ofrecida a la tecnología digital, la necesaria descentralización de la escuela como dispositivo unívoco del aprendizaje. En particular, los aportes de Pinto (2019) y Rivas (2019b) son reveladores para este fin. Es necesario procurar migraciones mentales que profesoras, profesores, directivos de la educación y familias debemos recorrer para pensar, en lugar de una escuela de las certezas, en una escuela de las preguntas y de las incertidumbres que ofrezcan soluciones creativas, que activen habilidades para insertar a niñas y niños en una sociedad de creatividad y colaboración en lugar de obediencia y docilidad.

Los sentimientos frente al aprendizaje reconstruidos por niñas y niños nos exhortan a la amplitud del binomio educación-tecnología digital. Selwyn (2016) nos recuerda que, si bien es innegable la capacitación en habilidades técnicas para participar del mercado laboral, no menos cierto es 
que el aprendizaje digital en toda su expresión significa un proceso de mayor calado, que implica la socialización y la subjetivación de niñas y niños que andan en formación del sentido de su existencia.

La pertenencia a grupos de interés en línea y la censura de contenidos abren una ventana para no cesar de investigar la nociva consecuencia de la limitación del efecto pares. Vivir aislado de otros, en definitiva, limita experiencias positivas de aprendizaje a niñas y niños, sin embargo, con la ventana digital parece superada esta limitación. Urge una investigación al respecto que, de ser transferida a la práctica escolar, podría en parte confrontar una de las consecuencias más nocivas del fenómeno segregador para el aprendizaje escolar.

Respecto a la identificación de los propios aprendizajes y la censura de contenidos ofrecimos resonancia a la voz de la niña y el niño en situación de segregación escolar. Voces que, por la tipología macrosocial de los estudios revisados en la primera sección, no aparecen. En este caso nos permitimos en el escenario microsocial hallar prácticas de autocuidado, reacciones ante la atrocidad de algunos contenidos digitales o los acuerdos entre pares para evitar los contenidos calificados como inadecuados. Nos sensibilizó el Ilamado de las niñas, que temen por su cuerpo, que sienten la presencia amenazante del otro en las redes, razón suficiente para no declinar en políticas que salvaguarden la seguridad digital. A la vez, navegamos con esperanza porque el proyecto activó la confianza de las niñas para narrar y actuar en torno a las áreas STEM, hasta hoy negadas y estigmatizadas como se aprecia en el informe Descifrar el Código (Unesco, 2019).

Enseñanza a la familia activa una línea de experimentación escolar para profesoras y profesores. Si bien, estudios como Murillo y Graña (2020b) estiman significativa la influencia del bajo capital cultural de las familias en la segregación escolar, no menos cierto es que las habilidades construidas por niñas y niños, pueden significar un interesante laboratorio escolar que, a través de la inversión de las responsabilidades de enseñanza de niñas y niños hacia sus familias aún con niveles de alfabetismo, revierta en alguna medida efectos de la segregación escolar en los aprendizajes. El aprendizaje digital de niñas y niños, sabiéndose profesores de sus padres, es una lección y un germen que requiere capitalización en un currículum escolar en migración.

Las fortalezas de este estudio recalcan en la posibilidad de explotar y transferir los invaluables hallazgos de la investigación macrosocial en torno a la segregación escolar socioeconómica, a la práctica escolar para tomar conciencia de una problemática de urgente intervención. Por tanto, 
la práctica escolar debe estar más abierta a la investigación, porque resulta ser el escenario propicio para el autoconocimiento, la concientización y la transformación de nuestras realidades.

El estudio resultó novedoso porque respondió a la necesidad de estudiar la relación entre segregación escolar socioeconómica y aprendizajes digitales, un terreno hasta el momento inexplorado, que deviene intervención desde la práctica investigativa situada. Además, podemos afirmar sin ingenuidad que el estudio aportó, como se confirman en las construcciones de niñas y niños, a estimular la confianza, a desarrollar la capacidad de narración y a soñar proyectos escolares digitales. Estas tres condiciones como lo advertimos en la revisión inicial son erosionadas por la situación desfavorecida que viven niñas y niños. Ratificamos aquí la vigencia de la propuesta de Tedesco (2012) de apostar por políticas de subjetividad, que transfieran a la singularidad de los contextos escolares, los estandarizados y homogéneos objetivos de las políticas educativas digitales.

Por último, mientras los investigadores escriben estas líneas, el mundo está sumido en una crisis sanitaria sin parangón. Entonces, sea esta la ocasión para insistir en la necesidad de recabar la noción de escuela. Ante esto, los relatos de niñas y niños en situación de segregación socioeconómica, evidencian la potencia que encierran los proyectos escolares digitales para expandir las posibilidades de aprendizaje, recrear los sentimientos frente al aprendizaje, permitir nuevas formas de asociación diferentes a la tradicional relación maestro-alumno y, por último, resituar a la familia como protagonista de los aprendizajes. Una vez más estamos llamados a ese notable esfuerzo por construir más escuela y menos aula (Fernández-Enguita, 2018).

Una limitación del presente estudio estriba en la imposibilidad de comparar los resultados con aprendizajes digitales de niñas y niños en situación escolar favorable. Lo que no deja de ser una oportunidad para explorar el fenómeno de la segregación hacia arriba, es decir, escuelas que concentran solo niños y niñas en condiciones socioeconómicas favorables. Para así, poder estimar con mayor asertividad los aprendizajes digitales construidos que son inherentes a la situación de segregación escolar socioeconómica.

Recomendamos a través de la investigación reactivar ánimos para actualizar la noción de condiciones de educabilidad (López y Tedesco, 2002), ahora al cariz del reto que significa la escuela digital. Pensar la educabilidad digital de niñas y niños significaría disponer predisposiciones, movilizar recursos disponibles, reconocernos en las limitaciones y crear proyectos escolares, a pesar de las crudas determinantes sociales, que devuelvan la confianza, la capacidad de narrar y soñar. Podría caber duda y preguntarnos: ¿para qué 
tomarnos la molestia de pensar la escuela digital? Con modestia respondemos: para algo no diferente que proporcionar mayor equidad social, basada en estimular oportunidades para la realización educativa de niñas y niños.

\section{Referencias}

Ball, S., Junemann, C. y Santori, D. (2017). Edu.net: Globalisation and Education Policy Mobility. Routledge.

Bellei, C. (2013). El estudio de la segregación socioeconómica y académica de la educación chilena. Estudios Pedagógicos, 39(1), 325-345. https://doi.org/10.4067/S0718$\underline{07052013000100019}$

Bonal, X. (2018). La política educativa ante el reto de la segregación escolar en Cataluña. UNESCO. https://bit.ly/37CqH6J

Bonal, X. y Zancajo, A. (2020). Elección de Escuela, Movilidad y Segregación Escolar del Alumnado Vulnerable en Barcelona. REICE. Revista Iberoamericana sobre Calidad, Eficacia y Cambio en Educación, 18(4), 197-218. https://doi.org/10.15366/ reice2020.18.4.008

British Council (2020). Programación para niños y niñas. Recuperado de https://bit.ly/360W8M9

Carretero, S., Vuorikari, R. y Punie, Y. (2017). DigComp 2.1 The Digital Competence Framework for Citizens With eight proficiency levels and examples of use. Recuperado de https:// bit.ly/3gf8VdD

Charmaz, K. (2006). Constructing grounded theory: A practical guide through qualitative analysis. SAGE.

CEN (2020). A common European Framework for ICT Professionals in all industry sectors. Recuperado de https://bit.ly/37SVaOH

Charmillot, S. y Felouzis, G. (2020). Modos de Agrupación de Estudiantes, Segregación y Desigualdades Educativas. Un Análisis Longitudinal de una Cohorte de Estudiantes en Suiza. REICE. Revista Iberoamericana sobre Calidad, Eficacia y Cambio en Educación, 18(4), 31-56. https://doi.org/10.15366/reice2020.18.4.002

Córdoba, C., Laborda, A. y Reyes, C. (2020). Preferencias de Elección de Escuela en Dos Casos de Alta Segregación Escolar. REICE. Revista Iberoamericana sobre Calidad, Eficacia y Cambio en Educación, 18(4), 325-344. https://doi.org/10.15366/ reice2020.18.4.013

Cortazar, L. y Taberner, P. A. (2020). La Incidencia del Programa Bilingüe en la Segregación Escolar por Origen Socioeconómico en la Comunidad Autónoma de Madrid: Evidencia a partir de PISA. REICE. Revista Iberoamericana sobre Calidad, Eficacia y Cambio en Educación, 18(4), 219-239. https://doi.org/10.15366/reice2020.18.4.009

Creswell, J. (2012). Educational research. Planning, conducting and evaluating quantitative and qualitative research. Pearson.

Denzin, N. K. y Lincoln, Y. S. (2005). The Sage handbook of qualitative research. SAGE. 
DQ Institute (2019). DQ Global Standards Report 2019 Common Framework for Digital Literacy, Skills and Readiness. Recuperado de https://bit.ly/33JFeMY

Dupriez, V. (2010). Methods of grouping learners at school. UNESCO.

Fernández-Enguita, M. (2018). Más escuela y menos aula. Madrid: Morata.

Ferrando, F., Hernández-Almeida, M., Oreiro, C., Seijas, M-N., y Urraburu, J. (2020). Evolución de la Segregación Socioeconómica en la Educación Pública de Uruguay. REICE. Revista Iberoamericana sobre Calidad, Eficacia y Cambio en Educación, 18(4), 143-169. https://doi.org/10.15366/reice2020.18.4.006

Friese, S. (2019). Qualitative Data Analysis with ATLAS.ti. SAGE.

Galtung, J. (1969). Violence, peace, and peace research. Journal of peace research, 6(3), 167 191. Recuperado de https://bit.ly/3qvl69U

Google for education. (2020). Applied digital skills. Recuperado de https://bit.ly/3mNBam9

Hass, P. M. (1992). Introduction: Epistemic Communities and International Policy Coordination. International Organization, 46(1), 1-35. REcuperado de https://bit.ly/2VEcd08

Ireson, J. y Hallam, S. (2001). Ability grouping in education. Sage: Paul Chapman Publishing.

ITU. (2019). Digital skills toolkit. Recuperado de https://bit.Iy/3mKn05r

Krüger, N. (2019). La Segregación por Nivel Socioeconómico como Dimensión de la Exclusión Educativa: 15 Años de Evolución en América Latina. Archivos Analíticos de Políticas Educativas, 27(8). https://doi.org/10.14507/epaa.27.3577

Krüger, N. (2020). Efectos Compañero en Contextos Escolares Altamente Segregados. REICE. Revista Iberoamericana sobre Calidad, Eficacia y Cambio en Educación, 18(4), 171-196. https://doi.org/10.15366/reice2020.18.4.007

Lisboa, T., Campelo, M., Macedo, F. y Lopes, D. (2020). Segregação Escolar e Desigualdades Educacionais no Início da Escolarização no Brasil. REICE. Revista Iberoamericana sobre Calidad, Eficacia y Cambio en Educación, 18(4), 77-96. https://doi. org/10.15366/reice2020.18.4.003

López, N. y Tedesco, J.C. (2002). Las condiciones de educabilidad de los niños y adolescentes en América Latina. IIPE-Unesco

Martínez-Garrido, C., Siddiqui, N. y Gorard, S. (2020). Estudio Longitudinal de la Segregación Escolar por Nivel Socioeconómico en Reino Unido. REICE. Revista Iberoamericana sobre Calidad, Eficacia y Cambio en Educación, 18(4), 123-141. https://doi. org/10.15366/reice2020.18.4.005

Microsoft. (2020). K-12 Computer Science Framework. Recuperado de https://k12cs.org

Mills, G. (2018). Action Research: A Guide for the Teacher Researcher. Pearson.

Murillo, F. J. (2016). Midiendo la Segregación Escolar en América Latina. Un Análisis Metodológico utilizando el TERCE. REICE. Revista Iberoamericana sobre Calidad, Eficacia y Cambio en Educación, 14(4), 33-60. https://doi.org/10.15366/reice2016.14.4.002

Murillo, F. J. (25 de septiembre 2019). Escuelas para pobres, escuelas para ricos. [Entrada de blog]. https://bit.ly/2VDloxg 
Murillo, F. y Carrillo, S. (2020). Una panorámica de la segregación escolar por nivel socioeconómico en educación primaria en Perú y sus regiones. Argumentos, 1(1), 7-31. https://doi. org/10.46476/ra.vi1.9

Murillo, F. J. y Duk, C. (2016). Segregación Escolar e Inclusión. Revista Latinoamericana de Educación Inclusiva, 10(2), 11-13. https://doi.org/10.4067/S0718$\underline{73782016000200001}$

Murillo, F. y Graña, R. (2020a). Una panorámica de la segregación escolar por nivel socioeconómico en Uruguay. Cuadernos de Investigación Educativa, 11(1), 15-35. https://doi. org/10.18861/cied.2020.11.1.2941

Murillo, F. y Graña, R. (2020b). ¿Segregación Escolar por Nivel Socioeconómico o por Nivel de Estudios de los Padres? REICE. Revista Iberoamericana Sobre Calidad, Eficacia y Cambio en Educación, 18(4), 9-29. https://doi.org/10.15366/reice2020.18.4.001

Murillo, F. J., y Martínez-Garrido, C. (2017a). Estimación de la magnitud de la segregación escolar en América Latina. magis, Revista Internacional de Investigación en Educación, 9(19), 11-30. https://doi.org/10.11144/Javeriana.m9-19.emse

Murillo, F. y Martínez-Garrido, C. (2017b). Segregación social en las escuelas públicas y privadas en América Latina. Educ. Soc., Campinas, 38(140), 727-750. https://doi. org/10.1590/ESO101-73302017167714

Murillo, F. J., Duk, C. y Martínez-Garrido, C. (2018). Evolución de la segregación socioeconómica de las escuelas de América Latina. Estudios pedagógicos, 44(1), 157-179. https:// doi.org/10.4067/S0718-07052018000100157

Murillo, F. J. y Martínez-Garrido, C. (2020). Magnitud de la Segregación escolar por nivel socioeconómico en España y sus Comunidades Autónomas y comparación con los países de la Unión Europea. Revista de Sociología de la Educación (RASE), 11(1), 37-58. https://doi.org/10.7203/RASE.11.1.10129

Nelson, M., Sahami, M. y Wilson, C. (7 de enero de 2016). Computing Leaders ACM, Code.org, and CSTA launch effort to guide educators and policymakers on K-12 computer science. [Entrada de blog]. https://bit.ly/3geUXZ9

OCDE (2012). Equity and Quality in Education: Supporting Disadvantaged Students and Schools. OCDE Publishing. Recuperado de https://bit.ly/2JOSwka

OCDE (2013). Results: Excellence Through Equity: Giving Every Student the Chance to Succeed, Vol. II. OCDE publishing. Recuperado de https://bit.ly/33PGb6q

OCDE (2016). Results: Excellence and Equity in Education, Vol. I. OECD Publishing. Recuperado de https://bit.ly/33PGgaK

OCDE (2019a). Envisioning the future of education and jobs. Trends, data and drawings. OECD Publishing. Recuperado de https://bit.ly/2VEAvYr

OCDE (2019b). Vectors of digital transformation. OECD Publishing. Recuperado de https://bit. ly/33Lwypf

OCDE (2019c). OECD Skills Outlook 2019. Thriving in a Digital World. OECD Publishing. Recuperado de https://bit.ly/3oxC2vT

OCDE (2020). Aprovechar al máximo la tecnología para el aprendizaje y la formación en América Latina. OECD Publishing. Recuperado de https://bit.ly/37EC3Hu

OXFAM (2015). Calculadora de la desigualdad. Recuperado de https://bit.ly/2Lcyr7Z 
Pinto, L. (2019). Rediseñar la escuela para y con las habilidades del siglo XXI. Fundación Santilla. Recuperado de https://bit.ly/2VUr47r

Ramírez, L., y Vazquez, E. (2020). Entendiendo los Cambios en la Segregación Escolar. Un Análisis en Base a Microdescomposiciones. REICE. Revista Iberoamericana sobre Calidad, Eficacia y Cambio en Educación, 18(4), 97-121. https://doi.org/10.15366/ reice2020.18.4.004

Rivas, A. (2019a). ¿Quién controla el futuro de la educación? Siglo XXI.

Rivas, A. (2019b). ¿Qué hay que aprender hoy? De la escuela de las respuestas a la escuela de las preguntas. Fundación Santillana. Recuperado de https://bit.ly/3osyT04

Rizvi, F. y Lingard, B. (2013). Políticas educativas en un mundo globalizado. Madrid: Morata.

Schmuck, R. A. (2006). Practical action research for change. Corwin Press.

Selwyn, N. (2016). Is technology good for education? John Wiley \& Sons.

SFIA Foundation. (2020). FIA view: Digital transformation view skills at a glance. Recuperado de https://bit.ly/3qpVZGu

Tedesco, J.C. (2012). Educación y justicia social en América Latina. Fondo de Cultura Económica.

UNESCO. (2015). Incheon declaration and framework for action for the implementation of Sustainable. Development Goal 4. UNESCO. Recuperado de https://bit.ly/3gluw4b

UNESCO. (2019). Descifrar el código: La educación de las niñas y las mujeres en ciencias, tecnología, ingeniería y matemáticas (STEM). UNESCO. Recuperado de https:// bit.ly/2VGD6kx

UNESCO Institute for Statistics. (2018). A global framework of reference on digital literacy skills for indicator 4.4.2. UNESCO. Recuperado de https://bit.ly/2JwNJ7c

UNICEF. (2017). El Estado Mundial de la Infancia 2017: Niños en un mundo digital. Recuperado de https://uni.cf/2Jx8lw6

Williamson, B. (2016). Digital methodologies of education governance. Pearson plc and the remediation of methods. European Educational Research Journal. 15(1), 34-53. https://doi.org/10.1177/1474904115612485

Williamson, B. (2018). Big data en educación. El futuro digital del aprendizaje, la política y la práctica. Madrid: Morata. 